\title{
Dual-Gate Polysilicon Nanoribbon Biosensors Enable High Sensitivity Detection of Proteins
}

\author{
I. Zeimpekis ${ }^{a \dagger}$, K. Sun ${ }^{\mathrm{a} \dagger}$, C. Hu ${ }^{\mathrm{a}}$, N. M. J. Ditshego ${ }^{\mathrm{a}}$, O. Thomas ${ }^{\mathrm{b}}$, M. R. R. de Planque ${ }^{\mathrm{a}}$, H. M. H. \\ Chong $^{\mathrm{a}}$, H. Morgan ${ }^{\mathrm{a}}$ and P. Ashburn ${ }^{\mathrm{a}}$
}

a. Zepler Institute, Electronics \& Computer Science, University of Southampton, Southampton, SO17 1BJ, UK.

b. Oxford Instruments Plasma Technology, Yatton, Bristol, BS49 4AP, UK.

$\dagger$ These authors have contributed equally.

Email: izk@ecs.soton.ac.uk, $\underline{\text { ks5@ecs.soton.ac.uk }}$

\begin{abstract}
We demonstrate the advantages of dual-gate polysilicon nanoribbon biosensors with a comprehensive evaluation of different measurement schemes for $\mathrm{pH}$ and protein sensing. In particular, we compare the detection of voltage and current changes when top- and bottom-gate bias is applied. Measurements of $\mathrm{pH}$ show that a large voltage shift of $491 \mathrm{mV} / \mathrm{pH}$ is obtained in the subthreshold region when the top-gate is kept at a fixed potential and the bottomgate is varied (voltage sweep). This is an improvement of 16 times over the $30 \mathrm{mV} / \mathrm{pH}$ measured using a top-gate sweep with the bottom-gate at a fixed potential. A similar large voltage shift of $175 \mathrm{mV}$ is obtained when the protein avidin is sensed using a bottom-gate sweep. This is an improvement of 20 times compared with the $8.8 \mathrm{mV}$ achieved from a topgate sweep. Current measurements using bottom-gate sweeps do not deliver the same signal amplification as when using bottom-gate sweeps to measure voltage shifts. Thus, for detecting a small signal change on protein binding, it is advantageous to employ a double-gate transistor and to measure a voltage shift using a bottom-gate sweep. For top-gate sweeps, the use of a dual-gate transistor enables the current sensitivity to be enhanced by applying a negative bias to the bottom-gate to reduce the carrier concentration in the nanoribbon. For $\mathrm{pH}$ measurements, the current sensitivity increases from $65 \%$ to $149 \%$ and for avidin sensing it increases from $1.4 \%$ to $2.5 \%$.
\end{abstract}

KEYWORDS: TFT, biosensor, nanorribon, nanowire, $\mathrm{pH}$ sensing, avidin, protein sensing

\section{Introduction}

Extensive research on ion sensitive field effect transistors has revealed the potential for label-free electronic detection of analytes in aqueous solutions [1]. The general sensing mechanism relies on the detection of the charge of the analyte when this binds to the surface or is in close proximity to the gate dielectric of the transistor biosensor. For proteins, the shortcoming arising from this sensing mechanism is that the surface potential changes caused by protein association are often small $[2,3]$ so current changes in the semiconductor are more difficult to detect than current changes resulting from ion association/dissociation.

To relieve this detection constraint, field effect transistor devices incorporating nanowires have been employed as sensors [4-7]. Nanowires have a large surface-to-volume ratio and hence their current output is more responsive to surface charges. They can be fabricated by both top-down [8] and bottom-up [9] technologies, with the top-down approach being more manufacturable, as it provides precise dimension and placement control. Nanowires fabricated in CMOS technologies have been widely studied and have been 
used to sense $\mathrm{pH}$ changes in solutions [10], proteins binding [11] on their surface, enzymatic reactions [12], DNA [13], and viruses [14]. However, their disadvantage is high cost due to the use of expensive Silicon-On-Insulator (SOI) wafers and advanced lithography.

The display panel industry has demonstrated that high quality and high yield systems can be manufactured using Thin Film Transistor (TFT) technologies. Therefore, these technologies have recently been explored as an approach for manufacturing very low cost disposable sensors [15]. In previous work [16], we demonstrated titration curves for the detection of cytokines using a polysilicon TFT nanowire technology. The results showed that the fullscale current change in the semiconductor channel for a complete titration of TNF- $\alpha$ was approximately $3 \%$. Cui et al. [17] described a bottom-up silicon nanowire sensor to measure the binding of streptavidin to a biotin modified surface. This device had a $\mathrm{pH}$ sensitivity of around $9 \%$ per $\mathrm{pH}$. The response at full saturation of streptavidin was $3 \%$, similar to our earlier work on TNF- $\alpha$. In a similar experiment Stern et al. [18] used a CMOS nanowire sensor with a $\mathrm{pH}$ sensitivity of about $500 \%$ per $\mathrm{pH}$ to detect streptavidin. This sensor's current changed $100 \%$ when its surface was saturated with streptavidin. In general, even sensors of extremely high $\mathrm{pH}$ sensitivity give only a moderate sensitivity in biosensing applications involving the binding of proteins.

A variety of measurement techniques have been reported by research groups, such as measuring a current change $[19,20]$ or a voltage shift $[21]$ by either using a top-gate [22] or a bottom-gate [23]. No clear view has been provided in the literature as to which of these techniques is the most effective. Dual-gate transistors [24-26] can accommodate a variety of these different measurement techniques. Furthermore, they offer the prospect of better control of the semiconductor channel and hence have the potential for a higher sensitivity, as required for reliable analyte detection and quantification. For example, it has been shown that $\mathrm{pH}$ sensing beyond the Nernst limit can be achieved using a dual-gate transistor [25]. There is therefore a strong motivation to further investigate dual-gate transistors for high sensitivity biosensing applications.

In this paper, we compare the benefits of top- and bottom-gate $\mathrm{pH}$ and protein sensing in thin film dualgate nanoribbon biosensors. The aim is to identify how different measurement methods such as top-gate versus bottom-gate sensing and current sensing versus voltage sensing can be used to maximize the signal change for a given sensor technology. We fabricated ultra-thin nanoribbon dual-gate biosensors as these are easy to manufacture using a simple top-down technology with surface-to-volume ratios that are competitive with many nanowire technologies. It is shown that top-gate sensing is better suited to the sensing of current changes, whereas bottom-gate sensing is better suited to the sensing of voltage changes. The insight provided by these measurements is then applied to the sensing of the avidin protein and it is shown that the use of a bottomgate provides a substantial amplification of the voltage shift.

\section{Methods}

Figure 1 shows a schematic view of the cross-section of a fabricated device, depicting the used materials. The sensors were fabricated junctionless (no highly doped source/drain) with a three-mask polysilicon process as described previously [15] on a Si substrate coated with a stack of $100 \mathrm{~nm}$ thermal $\mathrm{SiO}_{2}$ and $280 \mathrm{~nm}$ low pressure chemical vapour deposition (LPCVD) Si3N4. Figure 2 shows a microscope picture of a nanoribbon device. They consist of an array of 30, 40 um long and 2 um wide polysilicon nanoribbons covered by a $8.5 \mathrm{~nm}$ thermally grown $\mathrm{SiO}_{2}$. Additionally, a substantially large integrated $\mathrm{Ag} / \mathrm{AgCl}$ electrode was included in the sensor by $\mathrm{Ag}$ sputtering followed by chlorination to serve as a reference and top-gate electrode. SU8 windows such as the ones shown in figure 2 were patterned on the topside of the chips to expose the nanoribbon transistors to the liquid solutions under test. The effective length of the lightly doped nanoribbons, accessed through the SU8 window, is 30 um while the average doping concentration is roughly $5 \times 10^{17} \mathrm{~cm}^{-3}$. Bias was applied to the liquid (liquid gating) via the integrated reference electrode, which was also exposed through the SU8 window. A bottom-gate was created by contacting the bulk silicon using conductive epoxy bonding to a gold plated PCB. The sensor was wirebonded directly on a PCB to ensure reliable contacts, and all measurements were performed using SMA to Triaxial cables with an Agilent B1500A semiconductor device parameter analyzer.

Measurements of $\mathrm{pH}$ were carried out using universal buffer solutions $(0.1 \mathrm{M} \mathrm{NaCl}, 0.01 \mathrm{M}$ citric acid, $0.01 \mathrm{M}$ phosphoric acid, and $0.02 \mathrm{M}$ boric acid adjusted to $\mathrm{pH}$ values ranging from 3 to 9 by titration with a $1 \mathrm{M} \mathrm{NaOH}$ solution). The solutions were pipetted onto the sensor surface with the nanoribbons thoroughly washed with deionized water between measurements. 
Protein measurements were performed using syringe pumps connected to PDMS microfluidic channels (chemically bonded to SU8). The flow rate was set to $300 \mu \mathrm{l} / \mathrm{min}$. To enable avidin detection experiments, the sensors were first coated with the aminosilane (3aminopropyl)triethoxysilane (APTES, Sigma-Aldrich) through a chemical vapor deposition process in a Savannah Cambridge Nanotech ALD system. The APTES provided an amine-terminated surface for covalent immobilization of biotin. NHS-PEG4-BIOTIN (EZ-Link, Fisher Scientific UK Ltd) diluted in HEPES buffer (1 mM HEPES, and $0.67 \mathrm{mM} \mathrm{NaCl}$, titrated to $\mathrm{pH} 7.4$ with $1 \mathrm{M} \mathrm{NaOH}$ solution) at a concentration of 2 $\mathrm{mg} / \mathrm{ml}$ was introduced on the sensors for one hour. The biotin was subsequently replaced with a HEPES buffer solution and measurements were initiated. After initial settling, avidin (ImmunoPure, Fisher Scientific UK Ltd) (20 $\mathrm{nM}$ in $\mathrm{pH} 7.4$ HEPES buffer) was introduced and left on the surface for 10 minutes. The surface was then washed with clean buffer removing non-specifically bound avidin.

Two different biasing schemes were used for the sensing experiments; a) fixed bottom-gate potential with top-gate voltage sweep and b) fixed top-gate potential with bottom-gate voltage sweep. In both schemes, the source was grounded, the drain voltage $\left(V_{\mathrm{d}}\right)$ was kept constant at $100 \mathrm{mV}$, and the current through the drain $\left(I_{\mathrm{d}}\right)$ was measured.

All results were obtained using the measurement configuration shown in figure 3 , by continuous sweeps of subthreshold current/voltage characteristics using either the top $\left(V_{\text {top }}\right)$ or bottom $\left(V_{\text {bot }}\right)$ gate while monitoring the drain current $\left(I_{\mathrm{d}}\right)$; note that the subthreshold region is the region of interest [27]. A minimum of 50 sweeps were made for each protein sensing experiment. Using continuous sweeps provides a much better insight into the operation of the sensor when compared to fixed bias measurements or individual sweeps just before and after solution exchange. The continuous sweeps method gives a timeline of voltage and current changes for a wide range of gate bias conditions. A horizontal cutline of the subthreshold characteristics at a specific drain current gives the voltage as a function of time for this particular drain current, whereas a vertical cutline at a specific gate bias gives the current as a function of time for this gate bias. This data extraction process is repeated for every value of drain current and gate bias to provide a complete set of timeline results for all measurement conditions. This measurement method therefore provides excellent monitoring of the system stability and indicates the optimum range of conditions for measuring sensor performance for any given protein measurement.

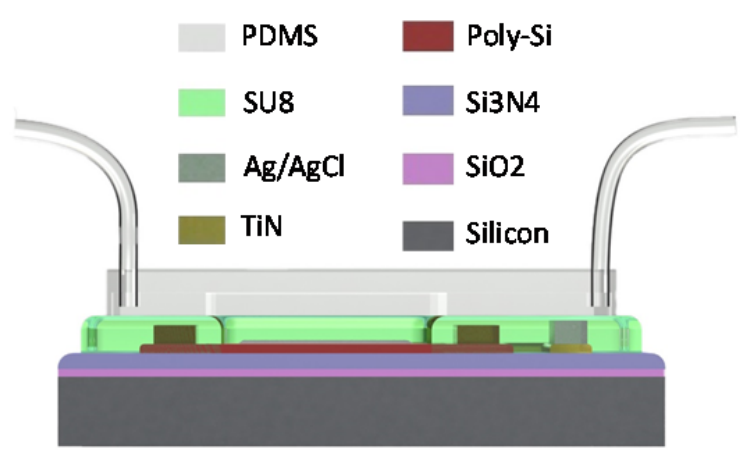

Figure 1. Cross-Section view of the fabricated device.

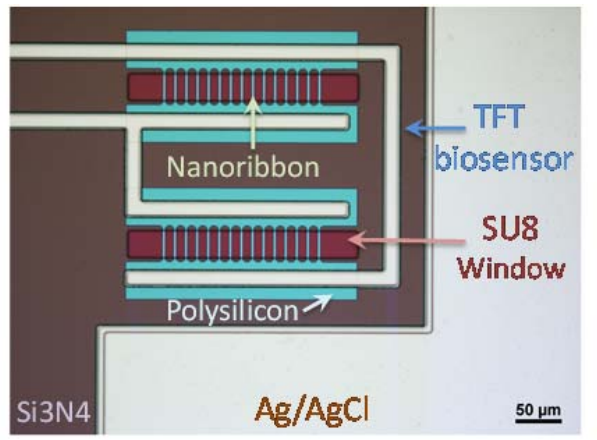

Figure 2. Microscope picture of a nanoribbon biosensor.

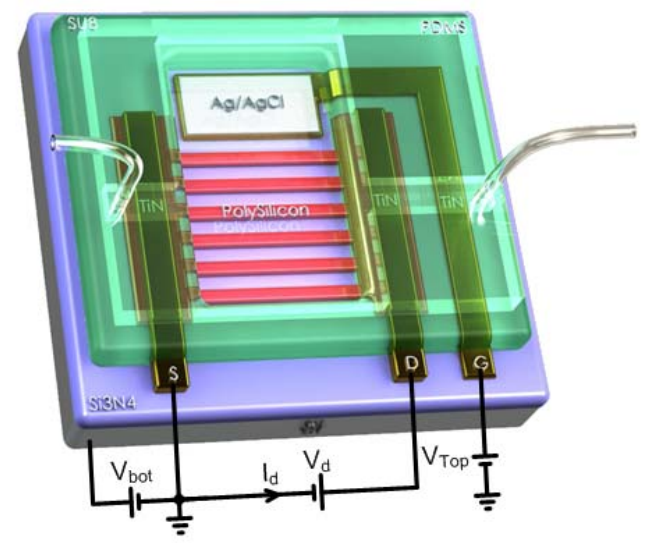

Figure 3. Schematic of the measurement configuration.

\section{Results and Discussion}

Figure 4 shows representative results from top-gate sweeps for a range of bottom-gate voltages between -5 and $-25 \mathrm{~V}$, using two buffer solutions of different $\mathrm{pH}$. This configuration allows for the characterization of the top-gate sensing at different bottom-gate biases. The I-V characteristics for the two different buffers are parallel in the subthreshold region for each bottom-gate voltage. Therefore, $\mathrm{pH}$ sensing can be performed by reading the voltage shift of the top-gate $\left(\Delta V_{\mathrm{T}, \mathrm{top}}\right)$ at a reference 
current level. It is apparent from figure 4 that an increasing negative bottom-gate bias causes the subthreshold slope to become steeper. This improvement in sensitivity is attributed to depletion of electrons in the n-type channel of the sensor by the application of the negative bottom-gate bias. Therefore, the subthreshold characteristics of the sensor can be tuned by depleting the channel through the bottom-gate to improve the subthreshold slope.

Figure 5 shows the current sensitivity as a function of top-gate voltage for different bottom-gate biases. Here current sensitivity is defined as the percentage change in the drain current for $\mathrm{pH} 9$ to $\mathrm{pH} 3$, normalized by the drain current at $\mathrm{pH} 9$. For each bottom-gate bias, the sensitivity varies with the potential of the top-gate with a peak at a specific top-gate voltage. There is therefore an optimum value of top-gate bias that gives maximum sensitivity, but this optimum value varies with bottom-gate bias. The continuous sweep measurement method provides the current sensitivity for different top-gate biases and therefore allows the current sensitivity to be maximized for a given set of measurement conditions or more generally for a given biosensor technology. Negative top-gate bias depletes the sensor by reducing the electron concentration in the channel, so a surface potential change on the gate causes a larger relative current change at the channel than a positive top-gate bias. The sensitivity reaches a maximum at the point where the channel is considered fully depleted. The graph reveals that for an increasing negative bottom-gate voltage the peak current sensitivity improves substantially from $65 \%$ at $V_{\text {bot }}=-5$ $\mathrm{V}$ to $149 \%$ at $V_{\text {bot }}=-25 \mathrm{~V}$.

Table 1 summarizes the key results obtained from the data shown in figure 4 and 5. It can be seen that the subthreshold slope, SS, improves for more negative bottom-gate voltages. When the subthreshold slope becomes steeper, the current differences arising from $\mathrm{pH}$ changes become larger, therefore the current sensitivity is improved. The fourth column of the table shows that regardless of biasing conditions, the top-gate voltage shifts $\Delta V_{\mathrm{T} \text {,top }}$ are always close to $30 \mathrm{mV} / \mathrm{pH}$. This is because surface potential changes resulting from $\mathrm{pH}$ value changes, have a Nernstian response and are not related to device bias or device electrical characteristics, but are solely determined by the gate dielectric material and the solution. Therefore, for a silicon dioxide surface the voltage shifts are limited to approximately $30 \mathrm{mV} / \mathrm{pH}$ [27]. On the contrary, current measurements from the top-gate are highly affected by the subthreshold performance of the sensor, hence highly sensitive current measurements were only achieved for specific biasing conditions that result in a steep subthreshold slope.

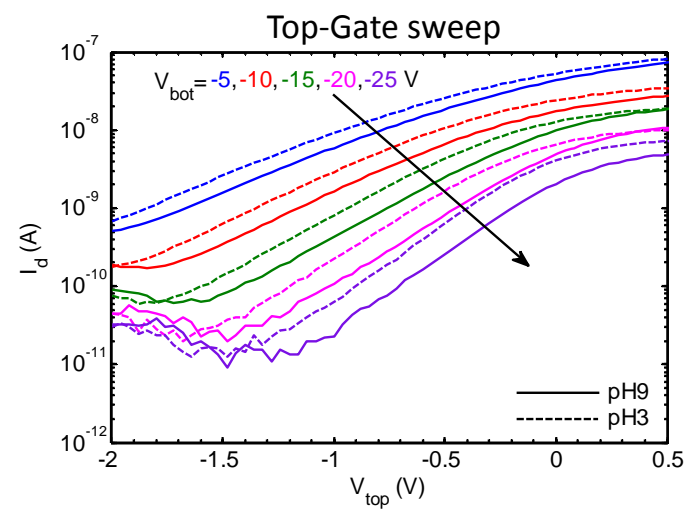

Figure 4. Drain current as a function of top-gate voltage for various bottom-gate voltages.

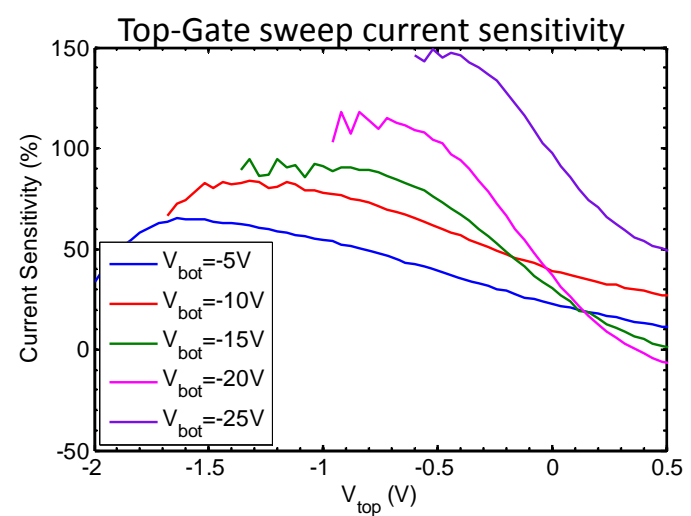

Figure 5. Current sensitivity against top-gate voltage for various bottom-gate voltages.

Table 1. Summary of electrical results obtained from top-gate sweeps for solutions with $\mathrm{pH}$ values of 3 and 9 .

\begin{tabular}{cccc}
\hline $\begin{array}{c}\text { Bottom-gate } \\
\text { voltage, }\end{array}$ & $\begin{array}{c}\text { Subthreshold } \\
\text { slope, } \\
V_{\text {bot }}(\mathrm{V})\end{array}$ & $\begin{array}{c}\text { Peak current } \\
\text { Sensitivity } \\
(\%)\end{array}$ & $\begin{array}{c}\text { Top-gate voltage } \\
\text { shift per } \mathrm{pH}, \\
\Delta V_{\mathrm{T}, \text { top }}(\mathrm{mV} / \mathrm{pH})\end{array}$ \\
\hline-5 & 886 & 65.5 & 29.8 \\
-10 & 750 & 83 & 31.8 \\
-15 & 600 & 95 & 30.6 \\
-20 & 585 & 118 & 30.9 \\
-25 & 500 & 149 & 32.3 \\
\hline
\end{tabular}

Figure 6 shows bottom-gate sweep results for different top-gate voltages ranging from 0.5 to $-1.5 \mathrm{~V}$. As the top-gate bias becomes more negative, the subthreshold slope becomes steeper. The plots of figure 6 show much larger bottom-gate voltage shifts between $\mathrm{pH}$ values compared to the top-gate plots of figure 4 .

The mechanism responsible for this result is the coupling of the potential change on the surface of the sensor through the two gate dielectrics $\left(C_{\mathrm{bot}}\right.$ and $\left.C_{\mathrm{top}}\right)$ 
and by a factor $\alpha_{\mathrm{SN}}$ with a value between 0 and 1 depending on the coupling through the channel material capacitance in the different operating regimes as discussed by Go et al. [28].

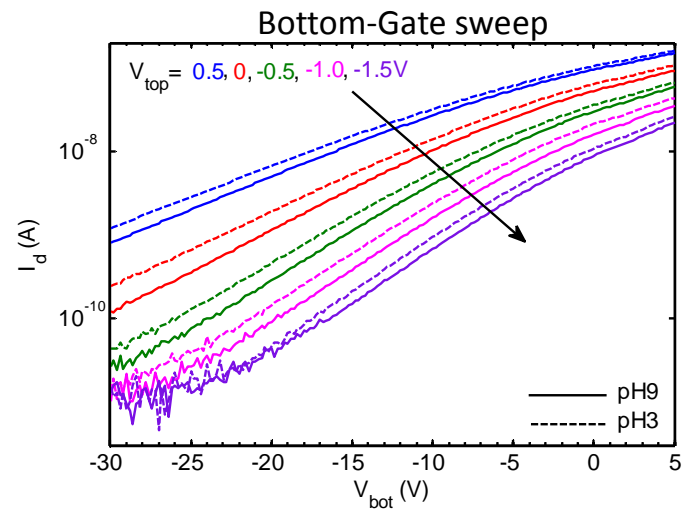

Figure 6. Drain current against bottom-gate voltage for different top-gate voltages.

This coupling amplifies the top-gate potential change $\left(\Delta V_{\mathrm{T}, \mathrm{top}}\right)$ which consequently results in an enhanced voltage shift for the bottom-gate sweeps $\left(\Delta V_{\mathrm{T}, \mathrm{bot}}\right)$, as shown in equation (1).

$$
\Delta V_{T, b o t}=\Delta V_{T, t o p} \frac{C_{t o p}}{C_{b o t}} \alpha_{S N}
$$

Table 2 summarizes the results from bottom-gate sweeps as extracted from the measurements of figure 6 . The first two columns show that the response of the bottom-gate voltage to $\mathrm{pH}$ varies with top-gate voltage. A maximum voltage shift of $491 \mathrm{mV} / \mathrm{pH}$ is achieved for $V_{\text {top }}=0 \mathrm{~V}$. This is a considerable improvement over the $30 \mathrm{mV} / \mathrm{pH}$ obtained for the top-gate sweeps. The third and fourth columns of Table 2 show that the current sensitivity does not track the subthreshold slope as was observed for the top-gate results. A maximum peak current sensitivity of $100 \%$ is achieved at $V_{\text {top }}=0 \mathrm{~V}$, which is beneficial because the maximum bottom-gate voltage shift and current sensitivity are met at the same top-gate voltage. The highest sensitivity for voltage sensing and current sensing therefore occurs at the same bias point. Clearly, the biggest benefit obtained when measuring using the bottom-gate is the amplification of the voltage shifts over those measured when using the top-gate. Essentially, bottom-gate sweeps amplify the voltage shift with a maximum amplification factor of 16, determined from Table 2. Bottom-gate sweeps are therefore, best suited to the measurement of voltage shifts in applications where the signals are very small, such as sensing of protein binding.

Equation (1) indicates that the amplification factor depends on the value of $\alpha_{\mathrm{SN}}$, which varies from 0 to 1 , depending on the region of operation of the transistor and the device structure [28]. Assuming a value of $\alpha_{\mathrm{SN}}$ of unity, equation (1) predicts an amplification factor of 30 for a top-gate bias of $0 \mathrm{~V}$, which compares with a measured value of 16 . In interpreting this result, it should be noted that the experimental results in Table 2 show a dependence of the bottom-gate voltage shift, and hence the amplification factor, on the applied top-gate voltage. This dependence is not predicted by equation (1), suggesting that further work is needed to develop a more complete theory.

Table 2. Summary of electrical results from bottom-gate sweeps for solutions with $\mathrm{pH}$ values of 3 and 9 .

\begin{tabular}{cccc}
\hline $\begin{array}{c}\text { Top-gate } \\
\text { voltage, } \\
V_{\text {top }}(\mathrm{V})\end{array}$ & $\begin{array}{c}\text { Bottom-gate voltage } \\
\text { shift per pH, } \\
\Delta V_{\text {T,bot }}(\mathrm{mV} / \mathrm{pH})\end{array}$ & $\begin{array}{c}\text { Subthreshold } \\
\text { slope, } \\
\text { SS (V/dec) }\end{array}$ & $\begin{array}{c}\text { Peak current } \\
\text { sensitivity } \\
(\%)\end{array}$ \\
\hline 0.5 & 338.6 & 13 & 50 \\
0 & 491 & 10 & 100 \\
-0.5 & 330.4 & 8.4 & 80 \\
-1 & 279.7 & 8.0 & 60 \\
-1.5 & 214.3 & 7.8 & 50
\end{tabular}

As mentioned in the introduction, the aim of this work is to identify the differences between top- and bottom-gate sensing, and then to harvest the advantages to achieve higher sensitivity in biosensing applications. The results presented above show that the current sensitivity of the sensor can be improved by using a topgate sweep, while applying a fixed bottom-gate voltage to deplete the channel. The current sensitivity can therefore be enhanced by carefully choosing the topand bottom- gate voltages, thereby enabling better protein sensing. Perhaps an even better approach is to sense a shift in the bottom-gate voltage by sweeping the bottom-gate voltage. The large amplification obtained using this measurement method should enable sensing of proteins, the association of which with the transistor surface does not result in a charge change as large as that resulting from a $\mathrm{pH}$ change of the solution.

To demonstrate the relative benefits of these two approaches, figure 7 and figure 8 show results on the sensing of avidin on a biotin-functionalized surface. Figure $7 \mathrm{a}$ and $\mathrm{b}$ show the drain current as a function of time for two different top-gate bias conditions. During this experiment, the bottom-gate was biased at $-15 \mathrm{~V}$, which offered the highest channel depletion with measurable current, and top-gate sweeps were performed in the subthreshold region. The variation in current with respect to time was extracted by multiple sweeps at the same conditions as described in the 
methods section. As presented in figure $7 \mathrm{a}$, at a top-gate voltage of $-1.2 \mathrm{~V}$ the current change after the introduction and wash of avidin is $2.5 \%$. In contrast, the change is $1.4 \%$ when the top-gate voltage is set to $-1 \mathrm{~V}$, as seen in figure $7 \mathrm{~b}$. The $\mathrm{pH}$ results in figure 5 showed that when the devices were biased at $V_{\text {bot }}=-15 \mathrm{~V}$ full depletion of their channel was achieved at $V_{\text {top }}=-1.2 \mathrm{~V}$, hence defining the point of maximum current sensitivity (for $V_{\mathrm{bot}}=-15 \mathrm{~V}$ ) when measuring using a top-gate. When the top-gate voltage drops to $-1 \mathrm{~V}$ the majority carrier concentration in the channel increases and therefore the current sensitivity drops.
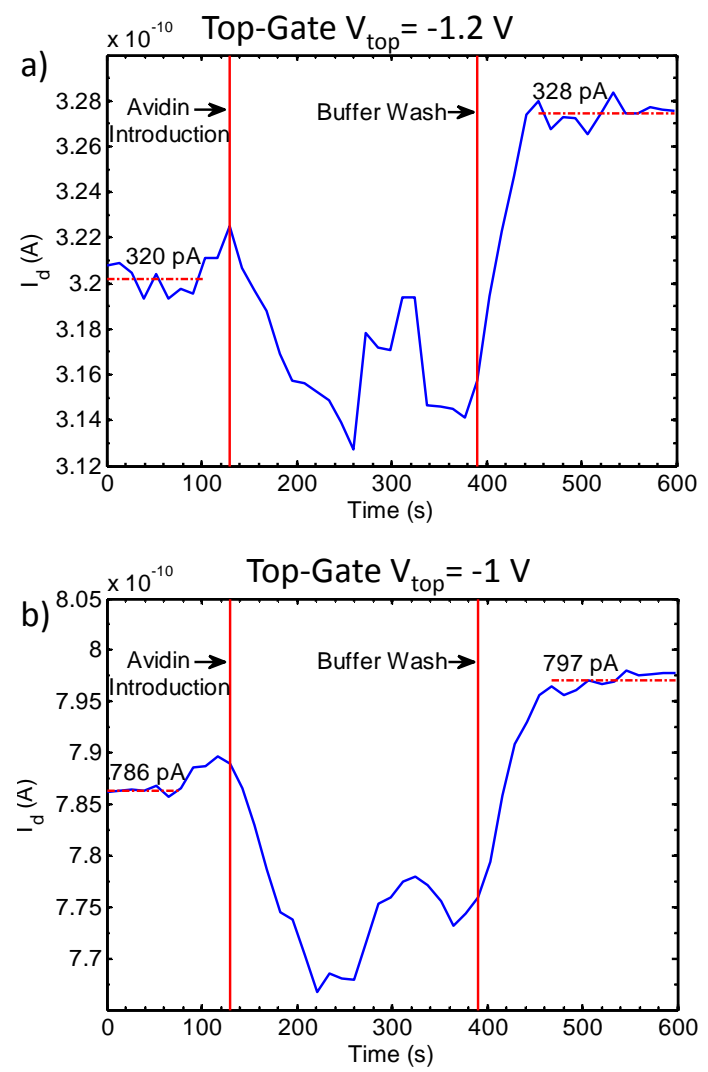

Figure 7. Avidin binding determined by measuring current changes from top-gate voltage sweeps $\left(V_{\text {bot }}=-15 \mathrm{~V}\right)$ at a) $V_{\text {top }}=-1.2 \mathrm{~V}$ and b) $V_{\text {top }}=-1 \mathrm{~V}$.

This result is important as it shows that protein binding induced current changes can be enhanced by tuning the voltages on the top- and bottom-gates. A double-gate sensor technology is therefore advantageous for protein sensing because it enables this tuning to maximize the current sensitivity. The double-gate nanoribbon technology used in this work provides a very simple method of realizing double-gate biosensors.

The experiment was repeated to evaluate voltage shift results from top- and bottom- gate sweeps. For comparison, figure $8 \mathrm{a}$ and $\mathrm{b}$ show the top- and bottom- gate results, plotted at the same voltage and current range. As the continuous sweeps allowed for, the reference currents were chosen to provide measurable results close to full depletion. For the bottom-gate sweeps shown in figure 8a the top-gate voltage was set to $0 \mathrm{~V}$ and the voltage shift was measured at a reference current of $500 \mathrm{pA}$. Figure $8 \mathrm{a}$ shows that avidin binding results in a voltage shift $\Delta V_{\mathrm{T}, \mathrm{bot}}$ of $175 \mathrm{mV}$. Figure $8 \mathrm{~b}$ shows equivalent results obtained using top-gate sweeps for the same experiment. In this case, avidin binding results in a voltage shift $\Delta V_{\mathrm{T} \text {,top }}$ of only $8.8 \mathrm{mV}$, at a reference current of $300 \mathrm{pA}$.
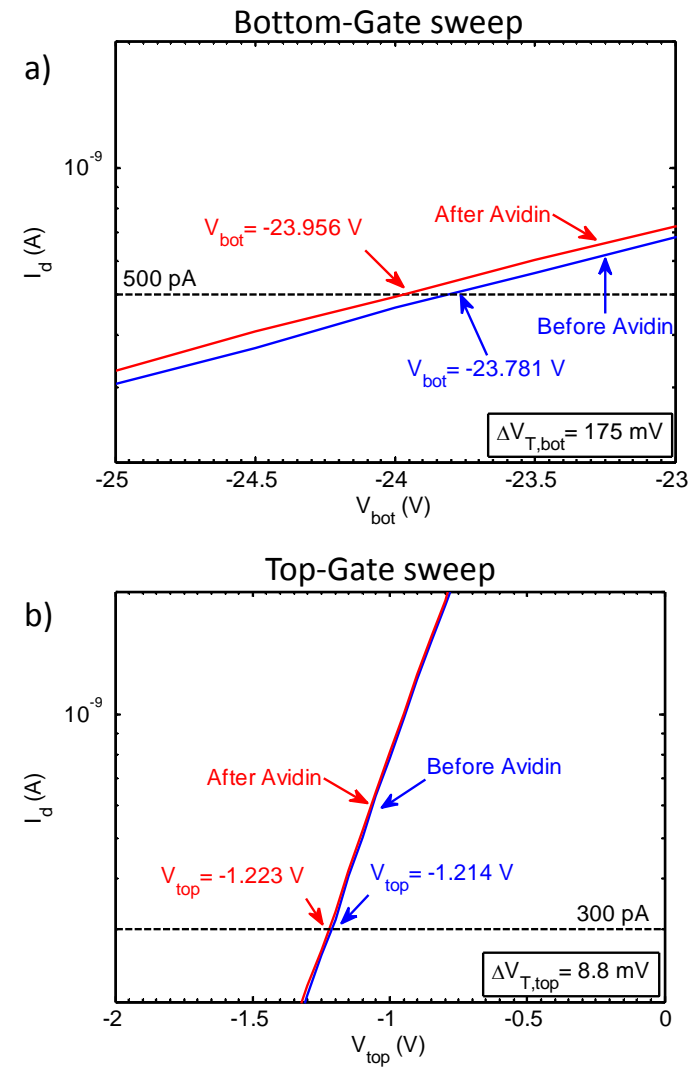

Figure 8. Avidin binding determined by measuring voltage changes by sweeping a) the bottom-gate and the b) the top-gate.

The measurement of avidin binding using a bottom-gate sweep has therefore resulted in a signal amplification of 20 times when compared to that obtained from a topgate sweep. As is seen from equation (1) to achieve such a high amplification a substantially thicker bottom-gate dielectric and consequently higher operating voltages are required when compared to the top-gate. Although, this can be limiting for some applications, the high amplification may be traded off for lower voltages by either using a thinner bottom-gate dielectric or by tuning the operating point of the sensor. Finally, a concern for every amplification scheme is the 
effective Signal-to-noise ratio (SNR). As discussed in [28] dual-gate sensors provide a higher signal and a lower noise when operated in the subthreshold region when compared to single-gated devices. Most importantly though, the large voltage output enables the use of less sophisticated instrumentation that is more applicable to point-of-care diagnostic platforms. In such systems the dominant noise source is the instrumentation and therefore dual-gated sensor can provide a higher SNR.

\section{Conclusions}

We have compared top- and bottom-gate measurements of $\mathrm{pH}$ and proteins using dual-gate nanoribbon TFT biosensors. For top-gate sweeps, $\mathrm{pH}$ and protein sensing measurements demonstrate that the application of a negative voltage to the bottom-gate significantly improves the current sensitivity. For $\mathrm{pH}$ sensing, an improvement in current sensitivity from $65 \%$ to $149 \%$ has been demonstrated and for protein sensing an improvement from $1.4 \%$ to $2.5 \%$. Thus for top-gate sweeps, a dual-gate transistor provides the additional flexibility of a bottom-gate where the bias can be tuned for optimum current sensitivity. Furthermore, the use of continuous sweeping of the topgate subthreshold characteristics provided a simple means of choosing the top-gate bias for optimum current sensitivity. For bottom-gate sweeps, $\mathrm{pH}$ and protein sensing experiments have shown that a large amplification of the signal change can be obtained if a voltage change is measured instead of a current change. For $\mathrm{pH}$ sensing, bottom-gate sweeps gave a 16 times higher voltage shift than top-gate sweeps and for protein sensing a 20 times higher voltage shift. This amplification of the signal change is very important for protein sensing because the signal changes obtained after protein binding are very small. In the current work, a $1.4 \%$ change in current sensitivity and an $8.8 \mathrm{mV}$ shift was originally observed for top-gate sweeps. These values are insufficient to enable complete measurement of a protein titration curve across the full range of protein concentrations. However, the 20 times amplification obtained by using bottom-gate sweeps gave a voltage shift of $175 \mathrm{mV}$, which significantly improves the sensitivity. Thus, a dual-gate transistor is highly desirable for protein sensing, as it allows sensing using bottom-gate sweeps providing the signal amplification necessary for sensitive detection.

\section{Acknowledgments}

The Authors declare no competing financial interest. The authors would like to acknowledge the Technology Strategy Board (TSB) and the Engineering and Physical Sciences Research Council (EPSRC: EP/K502327/1) for funding this work. We would also like to thank Sally Anderson, Gregory Gay, Ben Hadwen and Chris J. Brown of Sharp Laboratories Europe for many useful discussions. The data for this paper can be found at $10.5258 / \mathrm{SOTON} / 379790$

\section{References}

[1] Bergveld P 2003 Sens. Actuators B 88 1-20

[2] Nair P R, Alam M A 2008 Nano Lett. 8 12811285

[3] Chen R J, Choi H C, Bangsaruntip S, Yenilmez E, Tang X, Wang Q, Chang Y L, Dai H J 2004 Am. Chem. Soc. 126 1563-1568

[4] Chang H K, Ishikawa F N, Zhang R, Datar R, Cote R J, Thompson M E, Zhou C 2011 ACS Nano 5 9883-9891

[5] Gao Z, Agarwal A, Trigg A D, Singh N, Fang C, Tung C H, Fan Y, Buddharaju K D, Kong J 2007 Anal. Chem. 79 3291-3297

[6] Sarkar D, Liu W, Xie X, Anselmo A C, Mitragotri S, Banerjee K 2014 ACS Nano 8 3992-4003

[7] Gao A, Lu N, Dai P, Li T, Pei H, Gao X, Gong Y, Wang Y, Fan C. 2011 Nano Lett. 11 39743978

[8] Ditshego N M J, Sun K, Zeimpekis I, Ashburn P, de Planque M R R, Chong H M H 2015 Microelectron. Eng. 145 91-95

[9] Cui Y, Lieber C M, 2001 Science 291 851-853

[10] Chen Y, Xihua W, Erramilli S, Mohanty P, Kalinowski A 2006 Appl. Phys. Lett. 89

[11] Zheng G F, Patolsky F, Cui Y, Wang W U, Lieber C M, 2005 Nat. Biotechnol. 23 12941301

[12] Lu N, Dai P, Gao A, Valiaho J, Kallio P, Wang Y, Li T, 2014 ACS Appl. Mater. Inter. 6 20378-20384

[13] Hahm J I, Lieber C M 2004 Nano Lett. 4 51-54

[14] Zhang G J, Zhang L, Huang M J, Luo Z H H, Tay G K I, Lim E J A, Kang T G, Chen Y 2010 Sens. Actuators B 146 138-144

[15] Sun K, Zeimpekis I, Lombardini M, Ditshego N M J, Pearce S J, Kiang K S, Thomas O de Planque M R R, Chong H M H, Morgan H, 
Ashburn P 2014 IEEE Trans. Electron Devices 61 2170-2176

[16] Hakim M M A, Lombardini M, Sun K, Giustiniano F, Roach F D, Davies E P, Howarth H, de Planque M R R, Morgan H, Ashburn P 2012 Nano Lett. 12 1868-1872

[17] Cui Y, Wei Q, Park H, Lieber C M 2001 Science 293 1289-1292

[18] Stern E, Klemic J F, Routenberg D A, Wyrembak P N, Turner-Evans D B, Hamilton A D, LaVan D A, Fahmy T M, Reed M A 2007 Nature 445 519-522

[19] Zeimpekis I, Sun K, Hu C, Thomas O, de Planque M R R, Chong H M H, Morgan H, Ashburn P 2015 Nanoscale Res. Lett. 10

[20] Elfström N, Karlström A E, Linnros J 2008 Nano lett. 8 945-949

[21] Chen S, Bomer J G, Carlen E T, van den Berg A 2011 Nano Lett. 11 2334-2341

[22] Mu L, Droujinine I A, Rajan N K, Sawtelle S D, Reed M A 2014 Nano Lett. 14 5315-5322

[23] Li Y, Rajan N K, Routenberg D A, Modis Y, Reed M A 2012 Nat. Nanotechnol. 7 401-407

[24] Go J, Nair P R, Reddy B J, Dorvel B, Bashir R, Alam M A 2012 ACS Nano 6 5972-5979

[25] Knopfmacher O, Tarasov A, Fu W, Wipf M, Niesen B, Calame M, Schönenberger C 2010 Nano Lett. 10 2268-2274

[26] Ahn J H, Choi S J, Han J W, Park T J, Lee S Y, Choi Y K 2010 Nano Lett. 10 2934-2938

[27] Bousse L, De Rooij N F, Bergveld P 1983 IEEE Trans. Electron Devices 30 1263-1270

[28] Go J, Nair P R, Alam M A 2012 J. Appl. Phys. 112 